\title{
Perianal extragastrointestinal stromal tumor
}

\author{
Ayoung Kang', Sung Hwan Cho', Byung-Soo Park', Gyung Mo Son', Hyun Sung Kim', Jae-Joon Kim², Su Jin Kim², \\ Dong Hoon $\mathrm{Shin}^{3}$, Tae Un Kim ${ }^{4}$ \\ Departments of ${ }^{1}$ General Surgery, ${ }^{2}$ Internal Medicine, ${ }^{3}$ Pathology, and ${ }^{4}$ Radiology, Pusan National University Yangsan Hospital, Yangsan, Korea
}

An extragastrointestinal stromal tumor (EGIST) is a gastrointestinal stromal tumor that arises outside of the gastrointestinal tract. Most EGISTs are located in the omentum, mesentery, and retroperitoneum. The occurrence of an EGIST at the perianal region is very rare. Herein, we report our experience with EGISTs in the perianal area and review the literature. A 70-year-old man presented to our hospital with a 2-year history of anal discomfort. A pelvic magnetic resonance imaging scan showed a homogenous, well-defined, soft tissue density mass. The patient underwent mass excision, and the pathological examination confirmed that the mass was an EGIST. The size of the tumor was $4.3 \times 3.2$ $\mathrm{cm}$, and the mitotic count was 1 per 50 high-power fields. The tumor cells were immunohistochemically positive for KIT and CD34 but were negative for S-100 and alpha-smooth muscle actin. There were no other abnormal findings in the gastrointestinal tract; upon pathological review, this case was confirmed as perianal EGIST. Therefore, EGIST should be considered as a differential diagnosis of perianal masses.

Keywords: Extragastrointestinal stromal tumor, Gastrointestinal stromal tumor, Perianal neoplasm

\section{INTRODUCTION}

Gastrointestinal stromal tumors (GISTs) are the most common mesenchymal tumors in the gastrointestinal (GI) tract, and they are found to occur in approximately $1 \%$ of all GI tumors [1,2]. GISTs are common in the stomach $(60 \%-70 \%)$ and the small intestine $(25 \%-30 \%)$, whereas they are less frequent in the colon and rectum (5\%-15\%), duodenum (5\%), and esophagus ( $<2 \%)$ [3]. Some GISTs can be located outside of the GI tract, in which case they are referred to as extragastrointestinal stromal tumors (EG-

Received: Sep 22, 2020 Revised: Nov 26, 2020 Accepted: Dec 8, 2020

Correspondence to: Byung-Soo Park

Department of General Surgery, Pusan National University Yangsan

Hospital, 20 Geumo-ro, Mulgeum-eup, Yangsan 50612, Korea

Tel: +82-55-360-2124, Fax: +82-55-360-2154

E-mail: colonsurgeon@naver.com

ORCID: Ayoung Kang (https://orcid.org/0000-0002-5431-959X), Sung Hwan Cho (https://orcid.org/0000-0003-0451-5987), Byung-Soo Park (https://orcid.org/00000001-7782-5551), Gyung Mo Son (https://orcid.org/0000-0002-8861-6293), Hyun Sung Kim (https://orcid.org/0000-0002-7607-4597), Jae-Joon Kim (https://orcid. org/0000-0003-1226-2537), Su Jin Kim (https://orcid.org/0000-0003-3816-9664), Dong Hoon Shin (https://orcid.org/0000-0002-4980-9295), Tae Un Kim (https:// orcid.org/0000-0003-1017-6926)

Copyright (c) 2020 Korean Society of Surgical Oncology

This is an Open Access article distributed under the terms of the Creative Commons Attribution Non-Commercial License (http://creativecommons.org/licenses/by-nc/4.0) which permits unrestricted non-commercial use, distribution, and reproduction in any medium, provided the original work is properly cited.
ISTs). The incidence of EGISTs is reported to be approximately $1 \%$ of all GISTs [4]. Most EGISTs are located in the omentum, mesentery, and retroperitoneum, and EGISTs are very rare in the pancreas, prostate, abdominal wall, and perianal area [3]. Herein, we report our experience with an EGIST in the perianal area and review the literature. This study was approved by the Institutional Review Board of Pusan National University Yangsan Hospital (IRB No. 05-2020-198). The informed consent was waived.

\section{CASE REPORT}

A 70-year-old man presented to our hospital with a 2-year history of anal discomfort. He had a history of chronic aortic dissection (type A), thoracoabdominal aortic aneurysm and hypertension. There was no family history of cancer. Digital rectal examination revealed a movable and solid mass at a direction of 11 o' clock in the perianal area. Anoscopy showed no specific mucosal problems.

An abdominal and pelvic computed tomography (CT) scan revealed a homogenous, well-defined, soft tissue density mass with dimensions of $4.3 \times 2.5 \mathrm{~cm}$ at the right intersphincteric space (Fig. 1). A pelvic magnetic resonance imaging (MRI) scan also showed a homogenous, well-defined, soft tissue density mass with dimensions of $4.3 \times 3.9 \mathrm{~cm}$ with low signal intensity (SI) on T1-weighted image (T1WI), moderate high SI on T2WI, strong enhancement, and restricted diffusion (Fig. 2). The mass did not encroach the ex- 

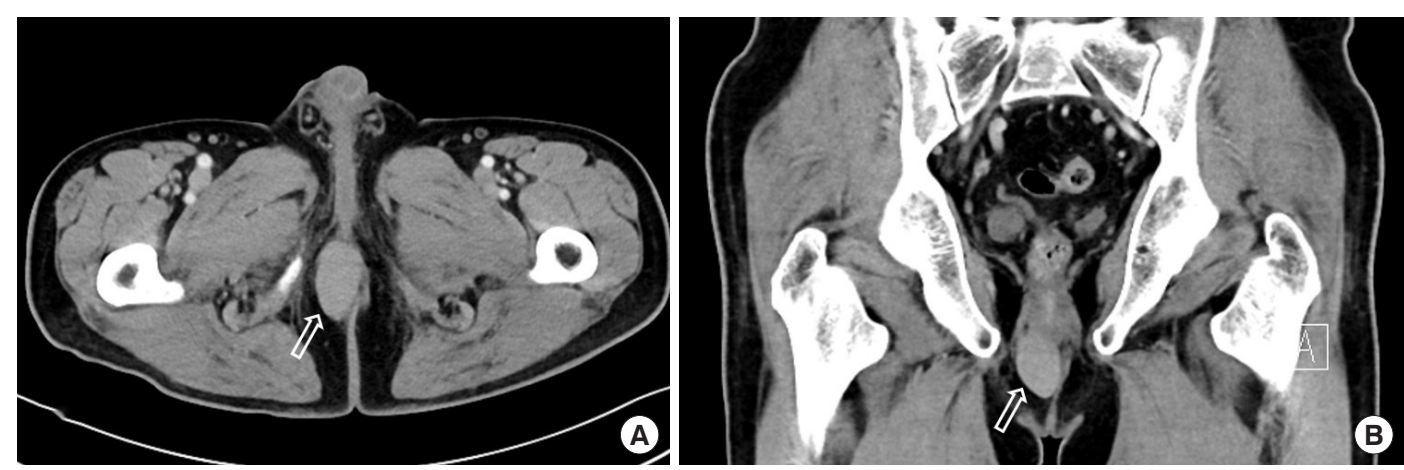

Fig. 1. Computed tomography abdomen and pelvis images. A $4.3 \times 2.5 \mathrm{~cm}$, homogenous, well-defined mass in the right intersphincteric space (arrow). (A) Transverse view. (B) Coronal view.
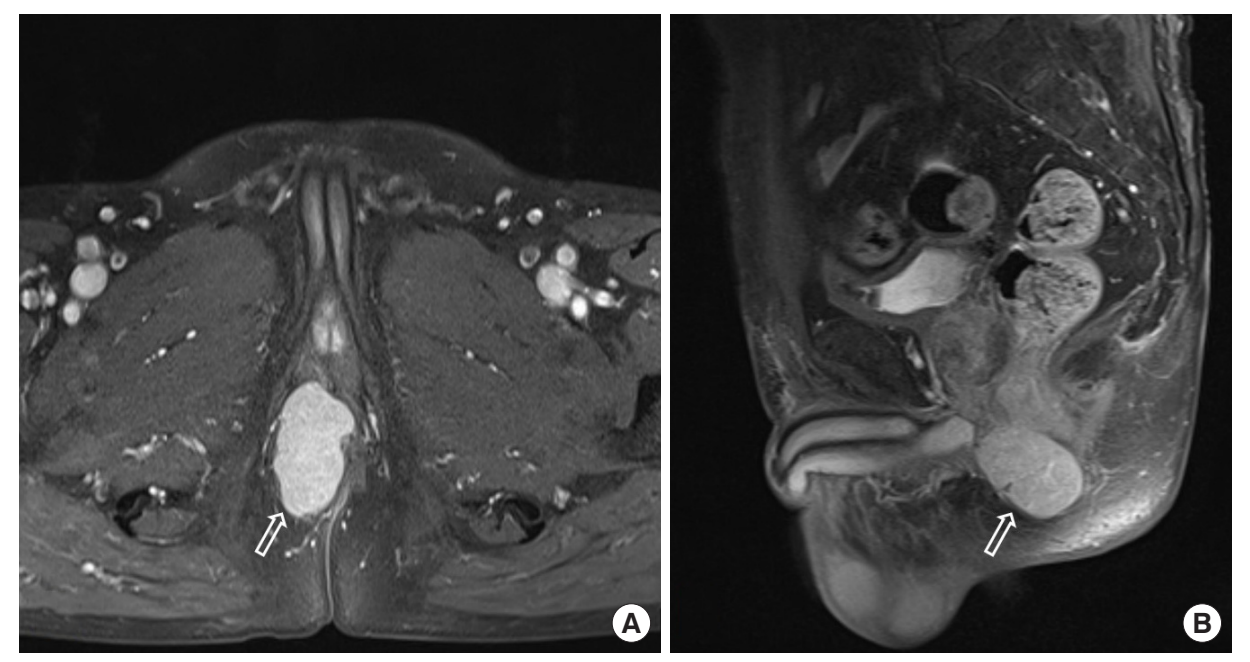

Fig. 2. Magnetic resonance pelvis images. A $4.3 \times 3.9 \mathrm{~cm}$, homogenous, well-defined mass in the right intersphincteric space (arrow). (A) Transverse view. (B) Sagittal view.

ternal anal sphincter with only extrinsic compression on the MRI. Ultrasonography-guided biopsy was performed, and the mass was histologically confirmed to be GIST. Esophagogastroduodenoscopy and colonoscopy were performed to exclude other primary sites, and no abnormal findings were observed in those examinations.

The patient underwent mass excision with the lithotomy position. The mass was located in the anterior lateral perianal region. A vertical incision was made according to the mass, $2 \mathrm{~cm}$ from the anal verge. In the operative field, the mass touched the skin of the anal verge interiorly; however, it did not involve the anal verge. The solid tumor was in contact with the external anal sphincter anterolaterally and was generally well demarcated. It involved a minor portion of the external anal sphincter $3 \mathrm{~cm}$ from the anal verge. Therefore, the mass could be removed with minor injury to the anal sphincter. The sphincter was repaired following mass excision. There was no involvement of the rectal wall. Pathological examination confirmed that the mass was an EGIST. The size of the tu- mor was $4.3 \times 3.2 \mathrm{~cm}$. The cut surface was yellow, white, and solid with focal hemorrhage. The tumor had mixed, spindle, and epithelioid cell types. The surgical margin was negative, although the tumor was very close to the surgical margin $(<1 \mathrm{~mm})$. The mitotic count was 1 per 50 high-power fields, and the tumor cells were immunohistochemically positive for KIT and CD34 but negative for S-100 and alpha-smooth muscle actin (Fig. 3). The Ki-67 labeling index was $<1 \%$. The patient had an uneventful postoperative course and was discharged 2 days after surgery. There was no recurrence of the tumor upon postoperative CT examination at 6 months. The patient did not complain of symptoms related to anal functions such as fecal incontinence or defecation difficulty at the 6-month follow-up. In addition, a digital rectal examination showed no change in anal sphincter function.

\section{DISCUSSION}

GISTs are tumors that arise from the interstitial cells of Cajal (ICC) 

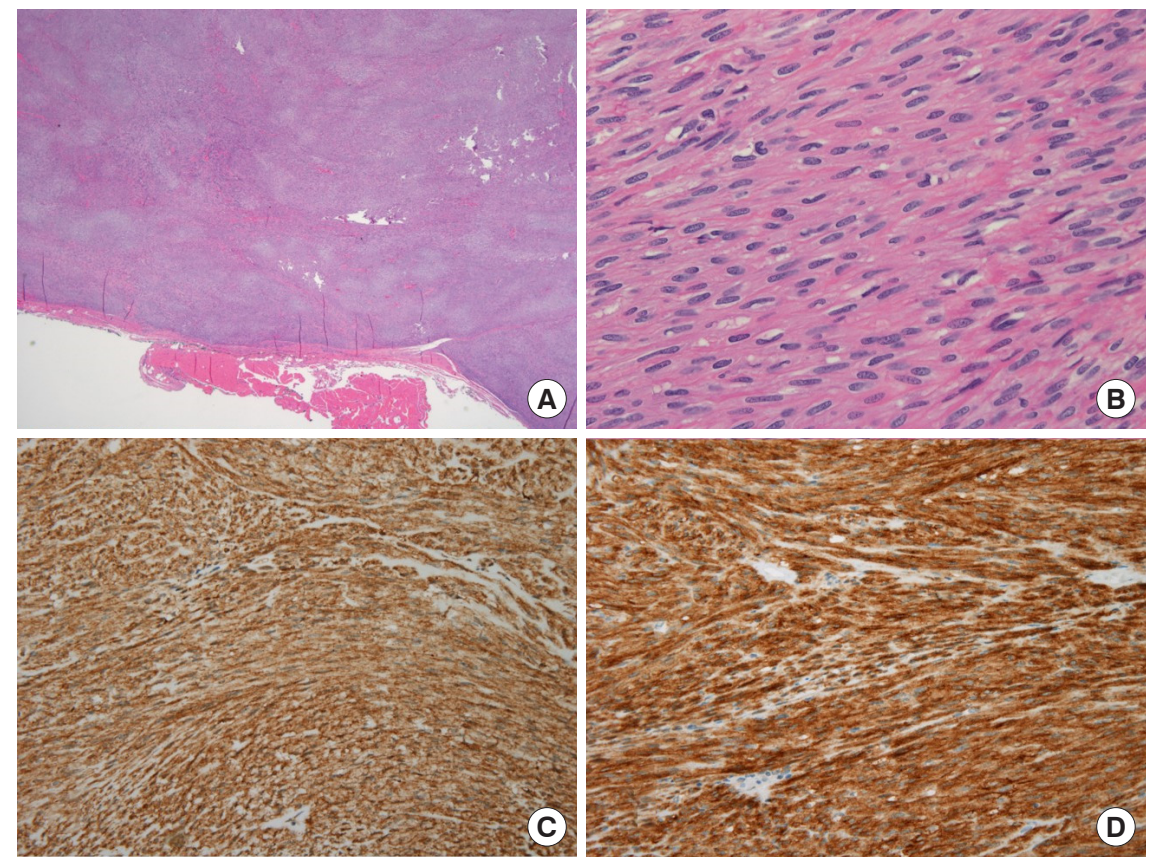

Fig. 3. Pathological and immunohistochemical findings. The tumor did not involve any bowel wall (H\&E: $A, \times 20 ; B \times 400)$. (C) Positive immunochemical staining for CD34 ( × 200). (D) Positive staining for KIT ( × 200).

[1]. Due to the fact that ICCs are more abundant in the stomach and small intestine, GISTs are more common in the stomach and the small intestine and less frequent in the esophagus and the rectum [5]. GISTs can arise outside of the GI tract, wherein they are known as EGISTs. There are several hypotheses on whether carcinogenesis can develop in sites without ICC [3]. First, because some interstitial Cajal-like cells are located in other organs outside of the GI tract, these cells may be the origin of EGISTs. Second, it is possible that EGISTs originate from pluripotent stem cells outside of the GI tract. Third, stromal tumors in the GI tract may spread to outside of the tract and can be identified as EGISTs.

EGISTs can be commonly located in the omentum and mesentery [3]. These areas are close to the stomach and the small bow$\mathrm{el}$, which are common sites of GISTs. Additionally, it is known that an omental EGIST has similar characteristics to a gastric GIST, whereas a mesenteric EGIST is similar to a GIST in the small bowel $[4,6]$. It seems that EGISTs may have a relationship with adjacent organs, although further studies are needed. Due to the fact that the incidence of perianal EGIST is extremely rare, there has been scarce information about its origin and characteristics. Based on previous reports, we suggest that a perianal EGIST may have similarities with a rectal GIST. More case reports are needed to confirm this hypothesis.

The incidence of EGISTs is at 1\% of all GISTs [4]. Agaimy and Wunsch [1] critically reviewed 200 cases with EGIST diagnoses, and they initially focused on the relationship between the tumors and muscularis propria of the bowel wall by reviewing surgical reports and pathological examinations. Surprisingly, most (>95\%) of the EGISTs were identified to actually involve the muscular layer of the bowel; thus, they were reassessed as being bowel GISTs. As a result, the actual incidence of EGISTs may be less frequent than has been previously reported. With this result, they presented the diagnostic criteria of EGIST, with some criteria required for basic GIST diagnoses and some criteria required for the exclusion of an apparent EGIST. The patient in our case report met the criteria properly.

Due to the fact that there has been no established prognostic factor for EGISTs, we have to draw analogies to that of GISTs [6]. The known factors of GISTs are size and proliferation indices, such as the mitotic rate, cellularity and location of the tumor. EGISTs may have a poorer prognosis than GISTs [3]. Due to the fact that the tumor itself is located outside of the GI tract, the symptoms may be presented at late stages and may be diagnosed at an advanced status of the disease, which can result in a poor prognosis. Some reports have suggested that the size of an EGIST does not affect the prognosis, and proliferation indices such as the mitotic rate were suggested as being the most important prognostic factor $[3,7,8]$. The biological behavior of EGISTs is known to differ between tumor locations [4]. For example, an omental EGIST is known to have a good prognosis (similar to gastric GIST), whereas a mesenteric EGIST has a poor prognosis (similar to a small bowel GIST). There have been no reports of the prognosis of a perianal EGIST, but it is estimated that a perianal EGIST may have a similar 
prognosis to that of a rectal GIST when referring to the characteristics of other EGISTs. Further studies on the prognosis of EGISTs are needed.

Localized resectable EGISTs can be cured with surgical resection as the treatment for GISTs [6]. Adjuvant treatment with imatinib mesylate is recommended for high-risk patients. In patients with an unresectable or metastatic EGIST, imatinib treatment and surgical resection can be helpful [9].

In summary, we experienced a very rare case of a perianal EGIST. Therefore, EGIST should be considered as a differential diagnosis of perianal masses. Further studies are needed to identify the clinical characteristics of a perianal EGIST and to apply them for clinical management.

\section{CONFLICT OF INTEREST}

No potential conflict of interest relevant to this article was reported.

\section{REFERENCES}

1. Agaimy A, Wunsch PH. Gastrointestinal stromal tumours: a regular origin in the muscularis propria, but an extremely diverse gross presentation: a review of 200 cases to critically re-evaluate the concept of so-called extra-gastrointestinal stromal tumours. Langenbecks Arch Surg 2006;391:322-9.

2. Miettinen M, Lasota J. Gastrointestinal stromal tumors: definition, clinical, histological, immunohistochemical, and molecular genetic features and differential diagnosis. Virchows Arch 2001;438:1-12.

3. Yi JH, Park BB, Kang JH, Hwang IG, Shin DB, Sym SJ, et al. Retrospective analysis of extra-gastrointestinal stromal tumors. World J Gastroenterol 2015;21:1845-50.

4. Miettinen M, Lasota J. Gastrointestinal stromal tumors: pathology and prognosis at different sites. Semin Diagn Pathol 2006;23:7083.

5. Graadt van Roggen JF, van Velthuysen ML, Hogendoorn PC. The histopathological differential diagnosis of gastrointestinal stromal tumours. J Clin Pathol 2001;54:96-102.

6. Kanamori K, Yamagata Y, Honma Y, Date K, Wada T, Hayashi T, et al. Extra-gastrointestinal stromal tumor arising in the lesser omentum with a platelet-derived growth factor receptor alpha (PDGFRA) mutation: a case report and literature review. World J Surg Oncol 2020;18:183.

7. Yamamoto H, Oda Y, Kawaguchi K, Nakamura N, Takahira T, Tamiya S, et al. c-kit and PDGFRA mutations in extragastrointestinal stromal tumor (gastrointestinal stromal tumor of the soft tissue). Am J Surg Pathol 2004;28:479-88.

8. Reith JD, Goldblum JR, Lyles RH, Weiss SW. Extragastrointestinal (soft tissue) stromal tumors: an analysis of 48 cases with emphasis on histologic predictors of outcome. Mod Pathol 2000;13:577-85.

9. Barros A, Linhares E, Valadao M, Goncalves R, Vilhena B, Gil C, et al. Extragastrointestinal stromal tumors (EGIST): a series of case reports. Hepatogastroenterology 2011;58:865-8. 